\title{
POPULATION DIVERSITY OF $n$-ALKANES IN THE NEEDLE CUTICULAR WAX OF RELICTS PINUS HELDREICHII AND P. PEUCE FROM THE SCARDO-PINDIC MOUNTAINS
}

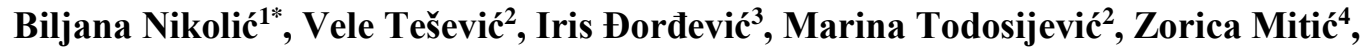 \\ Srdjan Bojović ${ }^{5}$, Petar D. Marin ${ }^{6}$ \\ ${ }^{1 *}$ Institute of Forestry, Kneza Višeslava 3, 11000 Belgrade, Serbia \\ ${ }^{2}$ Faculty of Chemistry, University of Belgrade, Studentski trg 16, P.O. Box 158, \\ 11000 Belgrade, Serbia \\ ${ }^{3}$ Faculty of Veterinary Medicine, University of Belgrade, Bulevar oslobođenja 18, \\ 11000 Belgrade, Serbia \\ ${ }^{4}$ University of Niš, Faculty of Sciences and Mathematics, Department of Biology and Ecology, \\ Višegradska 33, 18000 Niš, Serbia \\ ${ }^{5}$ Institute for Biological Research "Siniša Stanković”, University of Belgrade, \\ Boulevard Despota Stefana 142, 11060 Belgrade, Serbia \\ ${ }^{6}$ Faculty of Biology, Institute of Botany and Botanical Garden "Jevremovac", \\ University of Belgrade, Studentski trg 16, 11000 Belgrade, Serbia \\ smikitis@gmail.com
}

\begin{abstract}
The variability of $n$-alkanes in the needle cuticular wax of Pinus heldreichii and P. peuce in two natural populations from the Scardo-Pindic mountains was investigated for the first time. Gas chromatography (GC) and gas chromatography/mass spectrometry (GC-MS) analyses of two-year-old needles were performed using an Agilent 7890A GC equipped with an inert 5975C XL EI/CI mass spectrometer detector (MSD) and a flame ionization detector (FID) connected by a capillary flow technology 2-way splitter with make-up. An HP-5MS capillary column was used. $n$-Alkanes ranged from $\mathrm{C}_{19}$ to $\mathrm{C}_{33}$. In $P$. heldreichii the most abundant were $\mathrm{C}_{23}, \mathrm{C}_{25}$ and $\mathrm{C}_{27}\left(16.3,15.6\right.$ and $12.8 \%$ on average, respectively), while in $P$. peuce they were $\mathrm{C}_{29}, \mathrm{C}_{25}$, and $\mathrm{C}_{27}(16.5,15.3$ and $13.5 \%$ on average, resp.). Mid-chain and long-chain $n$-alkanes prevailed in both species. Principle component analysis (PCA) and Cluster analyses of five and six $n$-alkanes, respectively, showed divergence of the Scardo-Pindic populations from the Dinaric ones.
\end{abstract}

Keywords. Pinus heldreichii; P. peuce; $n$-alkanes, diversity, principle component analysis (PCA), cluster analysis

\section{ПОПУЛАЦИСКИ ДИВЕРЗИТЕТ НА $n$-АЛКАНИ ВО ВОСОК ОД КУТИКУЛИ НА ИГЛИЧКИ ОД РЕЛИКТНИ PINUS HELDREICHII И Р. PEUCE ОД СКАРДО-ПИНДИСКИТЕ ПЛАНИНИ}

За прв пат беше истражена варијабилноста на $n$-алкани во восок од кутикули на иглички од Pinus heldreichii и P. peисе во две природни популации од скардо-пиндискиот планински масив. Беа извршени гасна хроматографска (GC) и гасна хромоатографска/масено-спектрометриска (GC-MS) анализа, со употреба на Agilent 7890A GC опремен со инертен масен спектрометриски детектор (MSD) 5975C XL EI/CI и пламенен јонизациски детектор (FID), поврзани со капиларна проточна технологија со двонасочен сплитер со надоместување на гасови. Беше употребена капиларна колона HP-5MS. $n$-Алканите беа во опсегот од $\mathrm{C}_{19}$ до $\mathrm{C}_{33}$. Кај $P$. heldreichii најзастапени беа $\mathrm{C}_{23}, \mathrm{C}_{25}$ и $\mathrm{C}_{27}$ (просечно 16,3\%, 15,6 \% и 12,8 \%, соодветно), додека кај P. реисе тоа беа $\mathrm{C}_{29}, \mathrm{C}_{25}$, and $\mathrm{C}_{27}$ (просечно $16,5 \%, 15,3 \%$ и 13,5 \%, соодветно). И кај двата вида преовладуваа $n$-алкани со средни и долги низи. 
Анализата на главните компоненти (РСА) и кластер-анализата од соодветно пет и шест $n$-алкани покажа различност на скадро-пиндиските од динарските популации.

Клучни зборови: Pinus heldreichii; P. peисе; n-лакани; диверзитет; РСА; кластер-анализа

\section{INTRODUCTION}

Pinus heldreichii Christ. (Bosnian pine) is a Tertiary relict and a subendemic two-needle species. It grows naturally on the Balkan Peninsula and in southern Italy [1]. Several varieties, forms, and interspecific natural hybrids of $P$. heldreichii have been described [1].

Pinus peuce Griseb. (Macedonian pine) is a Tertiary relict and Balkan endemic five-needle species [1]. It grows naturally only on the Balkan Peninsula (on the high mountains of Bulgaria, North Macedonia, Montenegro, Serbia, Kosovo, Albania and the south of Greece).

These two pines grow alone or together on high mountains with different geologic substrata: limestones, silicates or schists [2,3]. In addition, owing to their decorative characteristics, they are often present in horticulture parks in Serbia.

$n$-Alkanes were used in fundamental $[4,5]$ or applied investigations [6] as well as in chemotaxonomic studies $[7,8]$.

Leaf $n$-alkanes studies have already shown population divergence in both pine species from the Dinaric mountains $[9,10]$. The aim of this study was to examine the population diversity of leaf $n$-alkanes of $P$. heldreichii and $P$. peuce originating from the Scardo-Pindic mountains and to compare them with previously examined populations originating from the Dinaric Alps $[9,10]$. To the best of our knowledge, this is the first report of population variability of $n$-alkanes of $P$. heldreichii and P. peuce originating from the Scardo-Pindic mountains.

\section{EXPERIMENTAL SECTION}

Plant Material. Two-year-old needles from two localities in the Scardo-Pindic mountains for both $P$. heldreichii (Mt. Baba -8 trees; Mt. Šara 16 trees) and P. peuce (Mt. Galičica - 10 trees; Mt. Šara - 14 trees) were collected from around the lower third of the unshaded tree crown at the end of the photosynthetic active season. Details about these populations have been already published [11, 12]. Twigs with needles were stored in polyethylene bags (with labels of the sample plot, date of collection, and age of the needles), and were transported to the freezer $\left(-20^{\circ} \mathrm{C}\right)$ in a hand fridge.

Extraction and isolation of needle wax. The concentrated extracts were chromatographed on a small-scale column using a Pasteur pipette filled with silica gel 60 previously activated at $120{ }^{\circ} \mathrm{C}$ [13]. Wax was obtained by elution with $5 \mathrm{ml}$ of $n$ hexane. Wax samples were stored at $-20{ }^{\circ} \mathrm{C}$ until further analysis.

Chemicals and reagents. Hexane (HPLC grade) and silica gel $60(0.2-0.5 \mathrm{~mm})$ were purchased from Merck (Darmstadt, Germany).

$G C$ and GC-MS analysis. Gas chromatography (GC) and gas chromatography/mass spectrometry (GC-MS) analyses were performed using an Agilent 7890A GC equipped with an inert 5975C XL $\mathrm{EI} / \mathrm{CI}$ mass spectrometer detector (MSD) and a flame ionization detector (FID) connected by a capillary flow technology 2-way splitter with make-up. An HP-5MS capillary column $(30 \mathrm{~m} \times 0.25 \mathrm{~mm} \times 0.25$ $\mu \mathrm{m})$ was used. The $\mathrm{GC}$ oven temperature was programmed from $60^{\circ} \mathrm{C}$ to $300{ }^{\circ} \mathrm{C}$ at a rate of $3{ }^{\circ} \mathrm{Cmin}^{-}$ ${ }^{1}$ and held for $10 \mathrm{~min}$. Helium was used as the carrier gas at $16.255 \mathrm{psi}$ (constant pressure mode). We used an auto-injection system (Agilent 7683B Series Injector) to inject $1 \mu \mathrm{l}$ of the sample. The sample was analyzed in the splitless mode. The injector temperature was $250{ }^{\circ} \mathrm{C}$ and the detector temperature $300^{\circ} \mathrm{C}$. MS data were acquired in EI mode with scan range of $30-550 \mathrm{~m} / z$, source temperature $230{ }^{\circ} \mathrm{C}$, and quadrupole temperature $150{ }^{\circ} \mathrm{C}$; solvent delay was 3 minutes.

Identification of n-alkanes. The components were identified based on their retention index and comparison with reference spectra (Wiley and NIST databases) as well as by the retention time locking (RTL) method and the RTL Adams data base. The retention indices were experimentally determined using the standard method of Van Den Dool and Kratz [14] involving retention times of $n$-alkanes injected after the sample under the same chromatographic conditions. The relative abundance of the $n$ alkanes (Tables 1,2) was calculated from the signal intensities of the homologues in the GC-FID traces.

Calculations of CPI and ACL values. The carbon preference index of total odd-numbered and even-numbered LNAs ( $\left.\mathrm{CPI}_{\text {total }}\right)$ was calculated by the formula of Mazurek and Simoneit [15]. The average chain length of total odd-numbered and evennumbered LNAs ( $\left.\mathrm{ACL}_{\text {total }}\right)$ was calculated by using the formula of Poynter and Eglinton [16]. In order to compare the obtained results with those from literature sources, $\mathrm{CPI}_{25-33}, \mathrm{CPI}_{20-36}, \mathrm{CPI}_{15-21}$, and $\mathrm{CPI}_{25-31}$ were also calculated by using the formula 
of Bray and Evans [17] as well as $\mathrm{ACL}_{23-35}$ by using the formula of Poynter and Eglinton [16]. Relative proportions of short-, mid-, and long-chain $n$-alkanes ( $n-\mathrm{C}_{18-20}, n-\mathrm{C}_{21-24}$ and $n-\mathrm{C}_{25-33}$, respectively) were also calculated [18].

Statistical analysis. The calculation of mean values $(\mathrm{X})$, standard deviations (SD), test for normality $\left(\chi^{2}\right.$ test), one-way analyses of variance (ANOVA), Levene's test, principal-component analyses (PCA) and cluster analysis were all carried out with the software Statgraphics Plus (version 5.0; Statistical Graphics Corporation, U.S.A.).

\section{RESULTS AND DISCUSSION}

Variability of n-alkanes. $n$-Alkanes in $P$. heldreichii and $P$. peuce from the Scardo-Pindic mountains ranged from $\mathrm{C}_{19}$ to $\mathrm{C}_{33}$ (Table 1). The range of $P$. heldreichii var. leucodermis grown in nurseries is narrower $\left(\mathrm{C}_{18}-\mathrm{C}_{31}\right)$ [8]. Some pines from the same subsection Pinus: P. mugo var. pumilio, P. sylvestris and $P$. sylvestris var. iberica had a range of $n$-alkanes different from $P$. heldreichii [8]. A wider range was found in $P$. mugo $\left(\mathrm{C}_{18}-\mathrm{C}_{33}\right)$ and a narrower range in $P$. nigra $\left(\mathrm{C}_{21}-\mathrm{C}_{33}\right)$ and $P$. thunbergii $\left(\mathrm{C}_{27}-\mathrm{C}_{29}\right)$.

In P. heldreichii needles from the Scardo-Pindic mountains, the most abundant $n$-alkanes were four odd-numbered: $\mathrm{C}_{23}, \mathrm{C}_{25}, \mathrm{C}_{27}$ and $\mathrm{C}_{29}(16.3 \%$, $15.6 \%, 12.8 \%$, and $11.1 \%$ on average, respectively) (Table 1), very often regarded as $\mathrm{C}_{\max }$ values (Table 2). Populations from Mt. Šara (II) had a higher content of $n$-alkanes $\mathrm{C}_{25}$ and $\mathrm{C}_{27}$ than the population from Galičica (I) (Fig.1). Populations of P. heldreichii from the Dinaric massif had similar amounts of $\mathrm{C}_{23}$, $\mathrm{C}_{27}$ and $\mathrm{C}_{25}(12.2 \%, 11.2 \%$ and $10.8 \%$ on average, resp.) [10]. The domination of several $n$-alkanes was also characteristic of $P$. mugo and $P$. pinea [8]. However, sometimes $P$. heldreichii var. leucodermis and some other pines were rich in only one $n$-alkane: $\mathrm{C}_{31}$ [8], $\mathrm{C}_{33}$ [19] or $\mathrm{C}_{29}$ [20].

Table 1

Chemical composition of $\mathrm{n}$-alkanes of Pinus heldreichii and Pinus peuce needles (\%)

\begin{tabular}{|c|c|c|c|c|c|c|c|c|c|c|c|c|}
\hline \multirow{5}{*}{ n-Alkanes } & \multicolumn{12}{|c|}{ Populations } \\
\hline & \multicolumn{4}{|c|}{ P. heldreichii } & \multicolumn{4}{|c|}{ P. peuce } & \multirow{2}{*}{\multicolumn{2}{|c|}{$\begin{array}{c}\text { P. heldreichii } \\
\text { Average }\end{array}$}} & \multirow{2}{*}{\multicolumn{2}{|c|}{$\begin{array}{l}\text { P.peuce } \\
\text { Average }\end{array}$}} \\
\hline & \multicolumn{2}{|c|}{ Mt. Galičica } & \multicolumn{2}{|c|}{ Mt. Šara } & \multicolumn{2}{|c|}{ Mt. Baba } & \multicolumn{2}{|c|}{ Mt. Šara } & & & & \\
\hline & \multicolumn{2}{|c|}{ I } & \multicolumn{2}{|c|}{ II } & \multicolumn{2}{|c|}{ I } & \multicolumn{2}{|c|}{ II } & \multicolumn{2}{|c|}{ I-II } & \multicolumn{2}{|c|}{ I-II } \\
\hline & $\mathbf{X}^{\mathrm{a}}$ & $S D^{\mathrm{b}}$ & $\mathbf{X}$ & $S D$ & $\mathbf{X}$ & $S D$ & $\mathbf{X}$ & $S D$ & $\mathbf{X}$ & $S D$ & $\mathbf{X}$ & $S D$ \\
\hline $\mathrm{C}_{19}$ & 0.36 & 0.07 & 0.35 & 0.18 & 0.39 & 0.13 & 0.51 & 0.11 & 0.36 & 0.15 & 0.45 & 0.13 \\
\hline $\mathrm{C}_{20}$ & 1.25 & 0.28 & 1.35 & 0.45 & 1.01 & 0.23 & 1.15 & 0.24 & 1.32 & 0.40 & 1.08 & 0.24 \\
\hline $\mathbf{C}_{21}$ & 4.34 & 0.63 & 4.50 & 0,87 & 3.05 & 0.73 & 3.51 & 0.60 & 4.44 & 0.78 & 3.28 & 0.70 \\
\hline $\mathbf{C}_{22}$ & 7.69 & 0.78 & 7.62 & 0.48 & 5.49 & 1.17 & 5.91 & 1.21 & 7.64 & 1.26 & 5.70 & 1.19 \\
\hline $\mathrm{C}_{23}$ & 16.61 & 2.36 & 16.11 & 1.28 & 13.47 & 2.18 & 13.45 & 1.40 & 16.29 & 1.69 & 13.46 & 1.80 \\
\hline $\mathrm{C}_{24}$ & 10.59 & 1.15 & 10.53 & 1.17 & 7.80 & 1.23 & 7.34 & 1.00 & 10.55 & 1.14 & 7.57 & 1.13 \\
\hline $\mathbf{C}_{25}$ & 14.04 & 1.55 & 16.44 & 1.61 & 14.66 & 1.88 & 16.00 & 2.02 & 15.60 & 1.95 & 15.33 & 2.04 \\
\hline $\mathrm{C}_{26}$ & 5.28 & 0.41 & 4.94 & 0.42 & 4.54 & 0.43 & 4.09 & 0.37 & 5.06 & 0.44 & 4.31 & 0.45 \\
\hline $\mathrm{C}_{27}$ & 11.80 & 1.39 & 13.34 & 2.18 & 12.50 & 1.46 & 14.47 & 1.12 & 12.80 & 2.05 & 13.48 & 1.62 \\
\hline $\mathrm{C}_{28}$ & 4.23 & 0.52 & 3.80 & 0.36 & 4.99 & 0.75 & 3.96 & 0.44 & 3.95 & 0.46 & 4.47 & 0.80 \\
\hline $\mathrm{C}_{29}$ & 11.51 & 2.33 & 10.91 & 1.16 & 17.59 & 4.13 & 15.45 & 1.73 & 11.12 & 1.63 & 16.52 & 3.30 \\
\hline $\mathrm{C}_{30}$ & 1.70 & 0.51 & 1.32 & 0.61 & 2.94 & 1.67 & 2.68 & 0.81 & 1.45 & 0.60 & 2.81 & 1.30 \\
\hline $\mathbf{C}_{31}$ & 5.54 & 1.80 & 5.16 & 1.21 & 5.61 & 2.26 & 6.31 & 1.81 & 5.29 & 1.41 & 5.96 & 2.04 \\
\hline $\mathrm{C}_{32}$ & 1.78 & 0.47 & 1.46 & 0.40 & 2.34 & 0.80 & 2.07 & 0.50 & 1.57 & 0.44 & 2.20 & 0.67 \\
\hline $\mathrm{C}_{33}$ & 3.30 & 1.78 & 2.16 & 0.42 & 3.62 & 0.92 & 3.09 & 0.78 & 2.56 & 1.20 & 3.36 & 0.88 \\
\hline
\end{tabular}

${ }^{\mathrm{a}}$ Mean value; ${ }^{\mathrm{b}}$ Standard deviation 


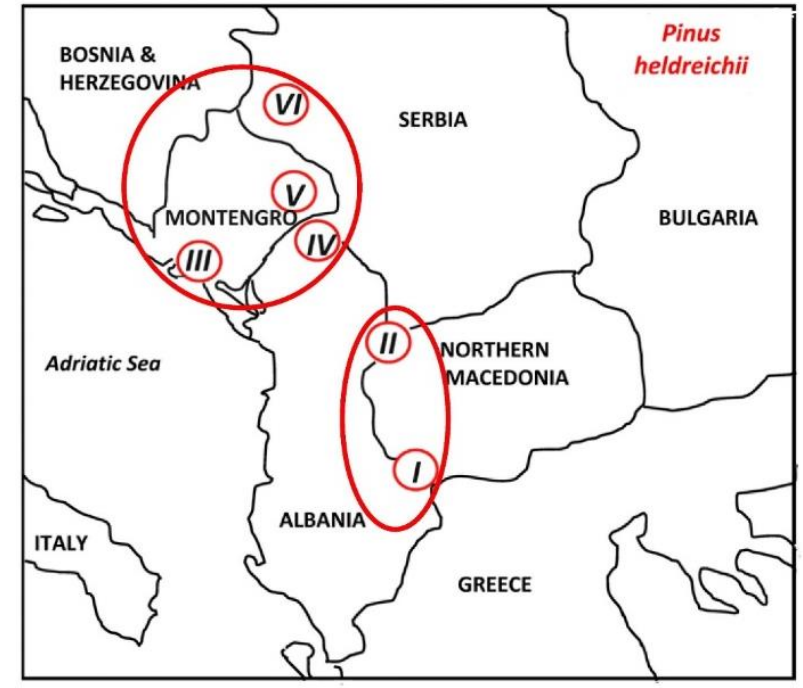

Fig. 1.Graphical illustration of Pinus heldreichii populations. Scardo-Pindic massif: Population I: Mt. Baba; Population II: Mt. Šara; Dinaric massif: Population III: Mt. Lovćen; Population IV: Mt. Zeletin; Population V: Mt. Bjelasica; Population VI: Mts. Zlatibor-Pešter;

In $P$. peuce needles, the most abundant were four odd-numbered $n$-alkanes: $\mathrm{C}_{29}, \mathrm{C}_{25}, \mathrm{C}_{23}$, and $\mathrm{C}_{27}$ $(16.5 \%, 15.3 \%, 13.5 \%$, and $13.5 \%$ on average, respectively) (Table 2). Populations from Mt. Šara (II) had a higher content of $n$-alkanes $\mathrm{C}_{25}$ and $\mathrm{C}_{27}$ than the population from Mt. Baba (I) (Fig.2). Populations of $P$. peuce from the Dinaric massif had similar amounts of $\mathrm{C}_{29}, \mathrm{C}_{25}, \mathrm{C}_{27}$, and $\mathrm{C}_{23}(15.5 \%, 11.1 \%, 10.6 \%$, and $10.5 \%$ on average, resp.) [21]. The domination of several $n$-alkanes was also characteristic of $P$. cembra var. glauca [21].

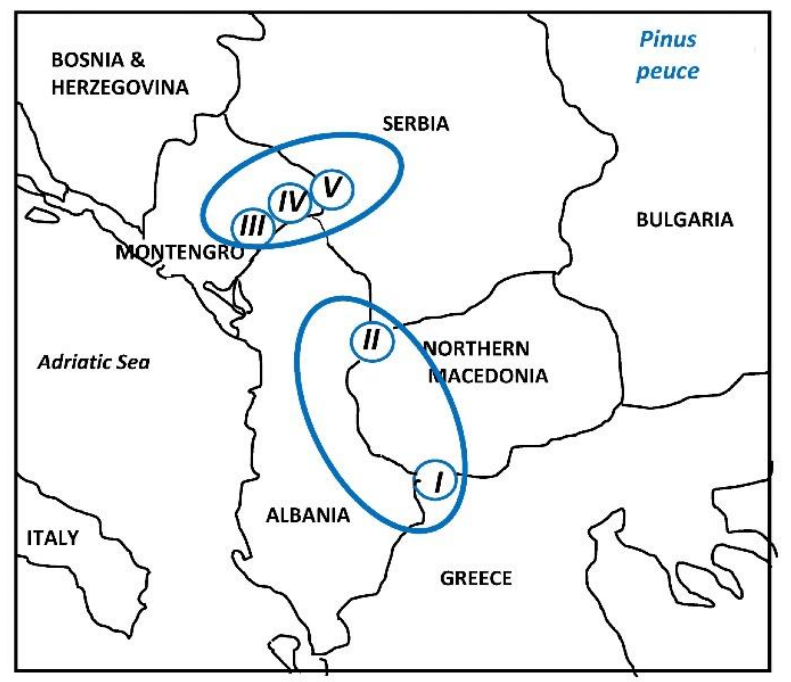

Fig. 2. Graphical illustration of Pinus peuce populations. Scardo-Pindic massif: Population I: Mt. Galičica; Population II: Mt. Šara; Dinaric massif: Population III: Mt. Zeletin; Population IV: Mt. Sjekirica; Population V: Mt. Mokra Gora.
Variability of CPIs and ACLs of n-alkanes. For the calculation of the amounts of long-chain $n$ alkanes (LNAs), i.e., CPIs and ACLs of $n$-alkanes of $P$. heldreichii and $P$. peuce from the Scardo-Pindic mountains (Table 2), the relative values from Table 1 were used. Maximum CPI values of long-chain $n$-alkanes of $P$. heldreichii from Scardo-Pindic mountains are $2.9\left(\mathrm{CPI}_{20-36}\right), 4.2\left(\mathrm{CPI}_{25-31}\right)$, and $4.2\left(\mathrm{CPI}_{25-33}\right)$ (Table 2). Short-chain $n$-alkanes $\left(\mathrm{CPI}_{15-21}\right)$ ranged from 1.7 to 2.8 (2.2 on average) and exhibited odd/even predominance (OEP) (because CPI $<1$ indicates EOP, CPI $>1$ denotes OEP) [18]. However, in our previous investigations, populations from Montenegro also showed OEP, while the ones from Serbia exhibited EOP. The CPI total $_{\text {of } P \text {. heldreichii }}$ from the Scardo-Pindic mountains is narrower but on average higher $(1.6-2.8 ; 2.1$ on average) than in the ones from the Dinaric massif $(0.8-3.1 ; 1.6$ on average) [18]. The $\mathrm{ACL}_{\text {total }}$ of $P$. heldreichii from the Scardo-Pindic mountains ranged from 24.9 to 26.5 (25.7 on average) and $\mathrm{ACL}_{23-35}$ ranged from 26.0 to 27.5 (26.4 on average) (Table 3 ). The $\mathrm{ACL}_{\mathrm{to}-}$ tal of $P$. heldreichii from the Dinaric mountains ranged from 20.9 to 26.5 (24.4 on average) and $\mathrm{ACL}_{23-35}$ ranged from 23.1 to 28.2 (26.1 on average) [9] (Table 2). The relative proportion of short-chain $n$-alkanes of $P$. heldreichii from the Scardo-Pindic mountains is small $\left(n-\mathrm{C}_{18-20}=1.7 \%\right)$, while longchain $n$-alkanes dominate over mid-chain ones (59.4 $\%$ and $39.0 \%$, resp.) (Table 2). Short-chain $n$-alkanes of $P$. heldreichii from the Dinaric mountains were significant $\left(n-\mathrm{C}_{18-20}=12.6 \%\right)$ and varied individually to a great extent (from 0.0 to 26.8) [9]. Slight domination of long-chain $\left(n-\mathrm{C}_{25-33}=49.6 \%\right)$ over mid-chain $n$-alkanes $\left(n-\mathrm{C}_{21-24}=37.9 \%\right)$ was found, too.

Maximum CPI values of long-chain $n$-alkanes of $P$. peuce from the Scardo-Pindic mountains are $2.2\left(\mathrm{CPI}_{20-36}\right), 3.0\left(\mathrm{CPI}_{25-31}\right)$, and $3.0\left(\mathrm{CPI}_{25-33}\right)$ (Table 2) which are equal or smaller than in $P$. peuce from the Dinaric massif $(2.3,1.2$, and 1.9, resp.) [10]. Short-chain $n$-alkanes $\left(\mathrm{CPI}_{15-21}\right)$ ranged from 1.0 to 4.3 (2.0 on average) and exhibited odd/even predominance (OEP) (because CPI $<1$ indicates EOP, CPI $>1$ denotes OEP). Short-chain $n$ alkanes $\left(\mathrm{CPI}_{15-21}\right)$ from the Dinaric massif ranged from 0.1 to 1.1 ( 0.6 on average) and exhibited even/odd predominance (EOP) [10]. The $\mathrm{ACL}_{\text {total }}$ of $P$. peuce from Scardo-Pindic mountains ranged from 24.9 to 26.6 (25.9 on average) and $\mathrm{ACL}_{23-35}$ ranged from 26.0 to 27.5 (26.6 on average) (Table 2 ). The $\mathrm{ACL}_{\text {total }}$ of $P$. peuce from the Dinaric mountains ranged from 0.9 to 5.5 (2.3 on average) and $\mathrm{ACL}_{23-35}$ ranged from 18.4 to 27.7 (25.7 on average) [10]. 
The relative proportion of short-chain $n$-alkanes of $P$. peuce from the Scardo-Pindic mountains is small $\left(n-\mathrm{C}_{18-20}=1.7 \%\right)$, while long-chain $n$-alkanes dominate over mid-chain ones $(61.8 \%$ and $35.0 \%$, resp.) (Table 3). Short-chain $n$-alkanes of $P$. peuce from the Dinaric mountains were also low $\left(n-\mathrm{C}_{18-20}=5.9 \%\right)$ and varied individually (from 0.0 to 20.1 ) [10]. Strong domination of long-chain $\left(n-\mathrm{C}_{25-33}=65.1 \%\right)$ over mid-chain $n$-alkanes $\left(n-\mathrm{C}_{21-24}=28.8 \%\right)$ was found.

Population diversity in regard to $\mathrm{n}$-alkane variability. For multi-variation principle-component analysis (PCA), five $n$-alkanes of 88 trees of $P$. heldreichii were selected (according to normal distribution, $\chi^{2}$, $P \geq 0.05$ and Levene's test, $P \geq 0.05$ ). In this study, previously examined populations from Montenegro and Serbia were added [9]. PCA revealed that the first two principal axes represent $68.7 \%$ of the total information (Fig. 3). Populations of P. heldreichii from the Scardo-Pindic mountains (I and II) overlapped and were clearly separated from populations Zeletin (IV) and Bjelasica (V). $n$-Alkanes which influenced the population diversity of $P$. heldreichii are presented in Table 3 .

Table 2

Analytical data for $\mathrm{n}$-alkanes in needles of Pinus heldreichii and Pinus peuce (\%)

\begin{tabular}{|c|c|c|c|c|c|c|c|c|c|c|c|c|c|}
\hline \multirow{2}{*}{\multicolumn{2}{|c|}{ Population }} & \multirow[t]{2}{*}{$\mathbf{C}_{\text {range }}$} & \multirow[t]{2}{*}{$\mathbf{C}_{\max }$} & \multirow[t]{2}{*}{ CPI ${ }_{\text {total }}{ }^{\mathrm{c}}$} & \multirow[t]{2}{*}{$\mathrm{CPI}_{25-33}{ }^{\mathrm{d}}$} & \multirow[t]{2}{*}{$\mathrm{CPI}_{20-36}{ }^{\mathrm{e}}$} & \multirow[t]{2}{*}{$\mathrm{CPI}_{15-21}{ }^{\mathrm{f}}$} & \multirow[t]{2}{*}{$\mathrm{CPI}_{25-31^{\mathrm{g}}}^{\mathrm{g}}$} & \multirow[t]{2}{*}{$A C L_{\text {total }}{ }^{h}$} & \multirow[t]{2}{*}{$\mathrm{ACL}_{23-35^{\mathrm{i}}}$} & $\begin{array}{l}\text { n-C } \mathbf{C}_{18-20} \\
\text { Short- } \\
\text { chain }\end{array}$ & $\begin{array}{c}\text { n-C } \\
\text { Mid-24 } \\
\text { chain }\end{array}$ & $\begin{array}{c}\text { n-C } \mathbf{C}_{25-33} \\
\text { Long- } \\
\text { chain }\end{array}$ \\
\hline & & & & & & & & & & & \multicolumn{3}{|c|}{ In $\%$ of total $n$-alkanes $\left(\mathrm{C}_{19-33)}\right.$} \\
\hline \multicolumn{14}{|c|}{ P. heldreichi } \\
\hline \multirow[t]{2}{*}{$\mathbf{I}$} & Range & 19-33 & $\begin{array}{l}23,25, \\
27,29\end{array}$ & $1.6-2.3$ & $2.1-3.4$ & $1.7-2.4$ & $1.9-2.7$ & $2.0-3.2$ & $25.2-26.5$ & $26.0-27.5$ & $1.3-2.1$ & $34.0-44.4$ & $53.5-64.6$ \\
\hline & Average & & 23 & 2.1 & 2.8 & 2.1 & 2.2 & 2.7 & 25.7 & 26.5 & 1.6 & 39.2 & 59.2 \\
\hline \multirow[t]{2}{*}{ II } & Range & 19-33 & $\begin{array}{l}25,23 \\
27,29\end{array}$ & $1.6-2.8$ & $2.4-4.2$ & $1.7-2.9$ & $1.7-2.8$ & $2.2-4.2$ & $24.9-25.9$ & $26.0-26.6$ & $1.0-2.9$ & $31.5-45.6$ & $51.6-67.3$ \\
\hline & Average & & 25 & 2.2 & 3.2 & 2.3 & 2.2 & 3.2 & 25.6 & 26.3 & 1.7 & 38.8 & 59.5 \\
\hline \multirow[t]{2}{*}{ I-II } & Range & 19-33 & $\begin{array}{l}23,25 \\
27,29\end{array}$ & $1.6-2.8$ & $2.1-4.2$ & $1.7-2.9$ & $1.7-2.8$ & $2.0-4.2$ & $24.9-26.5$ & $26.0-27.5$ & $1.0-2.9$ & $31.5-45.6$ & $51.6-67.3$ \\
\hline & Average & & 23 & 2.1 & 3.0 & 2.2 & 2.2 & 3.0 & 25.7 & 26.4 & 1.7 & 39.0 & 59.4 \\
\hline \multicolumn{14}{|c|}{ P.peuce } \\
\hline \multirow[t]{2}{*}{$\mathbf{I}$} & Range & 19-33 & $\begin{array}{l}29,25, \\
23,27\end{array}$ & $1.5-2.7$ & $2.5-4.1$ & $2.2-3.4$ & $0.1-0.9$ & $2.4-4.1$ & $25.8-27.1$ & $26.4-27.6$ & $0.8-2.0$ & $20.0-37.8$ & $60.3-79.2$ \\
\hline & Average & & 29 & 2.0 & 3.1 & 2.5 & 0.5 & 3.3 & 26.4 & 26.9 & 1.4 & 29.8 & 68.8 \\
\hline \multirow[t]{2}{*}{ II } & Range & 19-33 & $\begin{array}{l}25,29 \\
27,23\end{array}$ & $1.8-2.6$ & $2.8-4.9$ & $2.1-4.1$ & $0.2-1.1$ & $2.7-4.9$ & 25.9-26.6 & $26.4-27.3$ & $1.0-2.2$ & $26.5-34.2$ & $63.6-71.8$ \\
\hline & Average & & 25 & 2.1 & 3.6 & 2.8 & 0.6 & 3.4 & 26.2 & 26.8 & 1.7 & 30.2 & 68.1 \\
\hline \multirow[t]{2}{*}{ I-II } & Range & 19-33 & $\begin{array}{l}29,25 \\
27,23\end{array}$ & $1.6-2.8$ & $2.1-4.9$ & $1.7-4.1$ & $0.2-2.8$ & $2.0-4.9$ & $24.9-26.6$ & $26.0-27.5$ & $1.0-2.9$ & $26.5-45.6$ & $51.6-71.8$ \\
\hline & Average & & 29 & 2.1 & 3.3 & 2.5 & 1.4 & 3.2 & 25.9 & 26.6 & 1.7 & 35.0 & 61.8 \\
\hline
\end{tabular}

${ }^{\mathrm{c}} \mathrm{CPI} \mathrm{I}_{\text {total }}=\sum$ odd $\mathrm{Cn} n / \sum$ even $\mathrm{C} n$ [15], $\mathrm{C} n$ is the concentration of $n$-alkane containing $n$ carbon atoms;

${ }^{\mathrm{d}} \mathrm{CPI}_{25-33}=\left[\sum\left(\mathrm{C}_{25}-\mathrm{C}_{33}\right) \mathrm{odd} / \sum\left(\mathrm{C}_{24}-\mathrm{C}_{32}\right)\right.$ even $+\sum\left(\mathrm{C}_{25}-\mathrm{C}_{33}\right)$ odd $/ \sum\left(\mathrm{C}_{26}-\mathrm{C}_{34}\right)$ even $] / 2$ [17],

${ }^{\mathrm{e}} \mathrm{CPI}_{20-36}=\left[\sum\left(\mathrm{C}_{20}-\mathrm{C}_{36}\right)\right.$ odd $/ \sum\left(\mathrm{C}_{19}-\mathrm{C}_{35}\right)$ even $+\sum\left(\mathrm{C}_{20}-\mathrm{C}_{36}\right)$ odd $/ \sum\left(\mathrm{C}_{21}-\mathrm{C}_{37}\right)$ even $] / 2[17]$,

${ }^{\mathrm{f}} \mathrm{CPI}_{15-21}=\left[\sum\left(\mathrm{C}_{15}-\mathrm{C}_{21}\right)\right.$ odd $/ \sum\left(\mathrm{C}_{14}-\mathrm{C}_{20}\right)$ even $+\sum\left(\mathrm{C}_{15}-\mathrm{C}_{21}\right)$ odd $/ \sum\left(\mathrm{C}_{16}-\mathrm{C}_{22}\right)$ even $] / 2$ [17],

${ }^{\mathrm{g}} \mathrm{CPI}_{25-31}=\left[\sum\left(\mathrm{C}_{25}-\mathrm{C}_{31}\right)\right.$ odd $/ \sum\left(\mathrm{C}_{24}-\mathrm{C}_{30}\right)$ even $+\sum\left(\mathrm{C}_{25}-\mathrm{C}_{31}\right)$ odd $/ \sum\left(\mathrm{C}_{26}-\mathrm{C}_{32}\right)$ even $] / 2[17]$,

${ }^{\mathrm{h}} \mathrm{ACL}_{\text {total }}=\left(\sum \mathrm{C} n \times \mathrm{x} n\right) / \sum \mathrm{C} n[16]$,

${ }^{\mathrm{i}} \mathrm{ACL}_{23-35}=\left(23 \mathrm{xC}_{23}+25 \mathrm{xC}_{25}+27 \mathrm{xC}_{27}+29 \mathrm{xC}_{29}+31 \mathrm{xC}_{31}+33 \mathrm{xC}_{33}+35 \mathrm{xC}_{35}\right) /\left(\mathrm{C}_{23}+\mathrm{C}_{25}+\mathrm{C}_{27}+\mathrm{C}_{29}+\mathrm{C}_{31}+\mathrm{C}_{33}+\mathrm{C}_{35}\right)[16]$.

Ta ble 3

n-Alkanes (also quoted in Fig. 4) which influenced the diversity of Pinus heldreichii populations

\begin{tabular}{|c|c|c|c|c|c|}
\hline \multicolumn{6}{|c|}{ Pinus heldreichii } \\
\hline \multicolumn{3}{|c|}{ Scardo-Pindic mountains } & \multicolumn{3}{|c|}{ Dinaric mountains } \\
\hline Mt. Galičica & Mt. Šara & Mt. Lovćen & Mt. Zeletin & Mt. Bjelasica & Mt. Zlatibor-Pešter \\
\hline I & II & III & IV & $\mathrm{V}$ & VI \\
\hline $\mathrm{C}_{23}, 16.61 \%$ & $\mathrm{C}_{25}, 16.44 \%$ & $\mathrm{C}_{23}, 13.48 \%$ & $\mathrm{C}_{23}, 12.35 \%$ & $\mathrm{C}_{25}, 11.38 \%$ & $\mathrm{C}_{27}, 12.23 \%$ \\
\hline $\mathrm{C}_{25}, 14.04 \%$ & $\mathrm{C}_{23}, 16.11 \%$ & $\mathrm{C}_{28}, 3.96 \%$ & & $\mathrm{C}_{27}, 11.23 \%$ & $\mathrm{C}_{28}, 4.13 \%$ \\
\hline $\mathrm{C}_{27}, 11.80 \%$ & $\mathrm{C}_{27}, 13.34 \%$ & & & & \\
\hline
\end{tabular}

" - original data ," - literature data [9] 


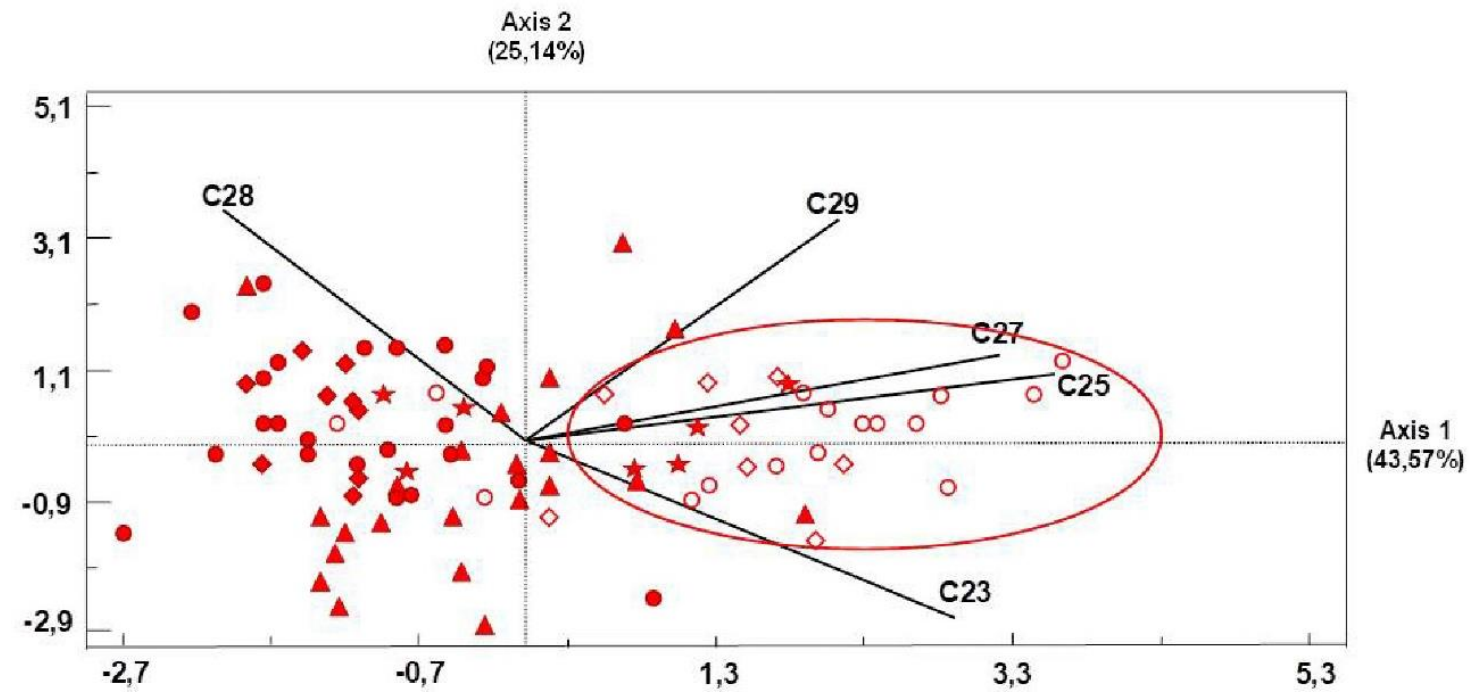

Fig. 3. Principle-component analysis of five selected $n$-alkanes isolated from 88 Bosnian pine-tree samples from six populations. $n$-Alkanes: $\mathrm{C}_{23}, \mathrm{C}_{25}, \mathrm{C}_{27}, \mathrm{C}_{28}$, and $\mathrm{C}_{29}$. Population I: Mt. Baba $\diamond$; Population II: Mt. Šara $\circ$; Population III: Mt. Lovćen $\boldsymbol{\Delta}$; Population IV: Mt. Zeletin $\downarrow$; Population V: Mt. Bjelasica •; Population VI: Mts. Zlatibor-Pešter $\star$

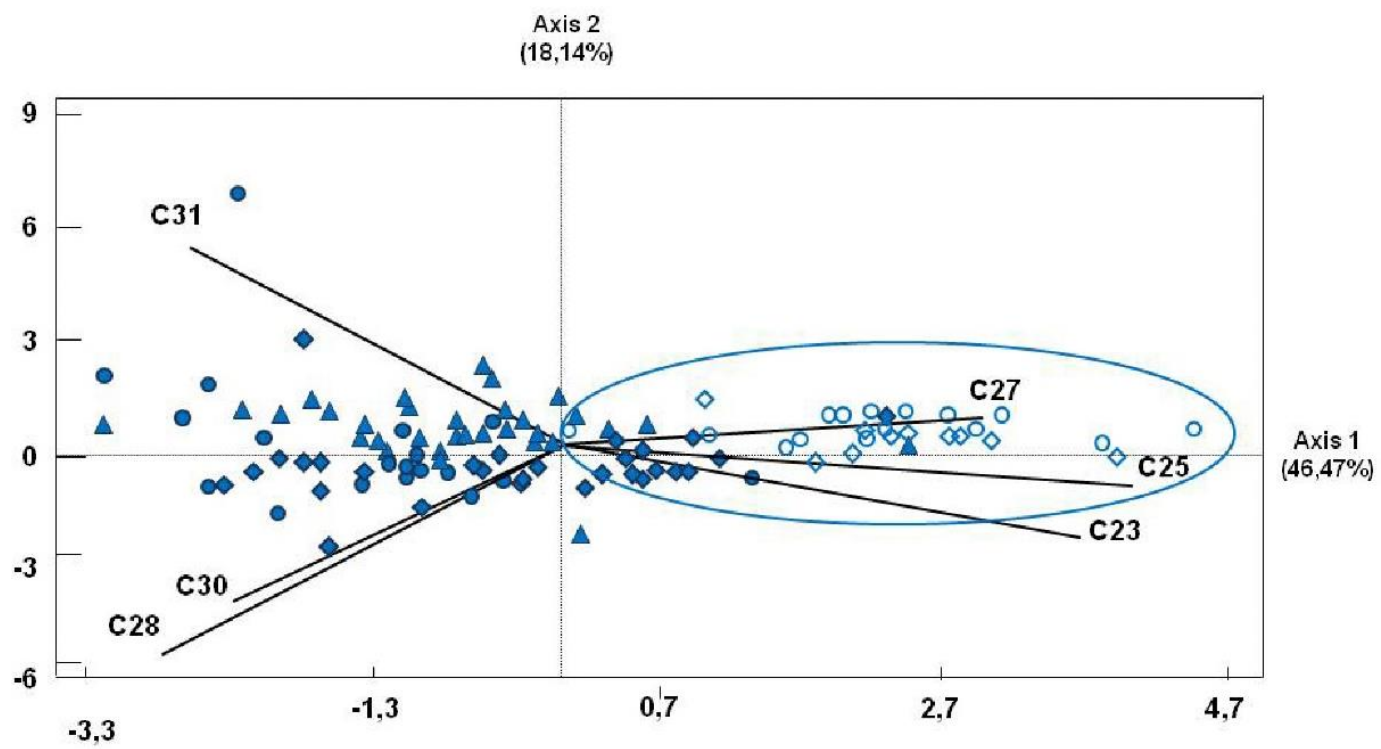

Fig. 4. Principle-component analysis of five selected n-alkanes isolated from 109 Macedonian pine-tree samples from five populations. $n$-Alkanes: $\mathrm{C}_{23}, \mathrm{C}_{25}, \mathrm{C}_{27}, \mathrm{C}_{28}, \mathrm{C}_{30}$ and $\mathrm{C}_{31}$. Population I: Mt. Galičica $\diamond$; Population II: Mt. Šara $\circ$; Population III: Mt. Zeletin $\boldsymbol{\Delta}$; Population IV: Mt. Sjekirica $\diamond$; Population V: Mt. Mokra Gora $\bullet$

T a ble 4

n-Alkanes (also quoted in Fig. 5) which influenced the diversity of Pinus peuce populations

\begin{tabular}{|c|c|c|c|c|}
\hline \multicolumn{5}{|c|}{ Pinus peuce } \\
\hline \multicolumn{2}{|c|}{ Scardo-Pindic mountains } & \multicolumn{3}{|c|}{ Dinaric mountains *" } \\
\hline Mt. Baba & Mt. Šara & Mt. Zeletin & Mt. Sjekirica & Mt. Mokra Gora \\
\hline $\mathrm{I}$ & II & III & IV & $\mathrm{V}$ \\
\hline $\mathrm{C}_{29}, 17.59 \%$ & $\mathrm{C}_{25}, 16.00 \%$ & $\mathrm{C}_{29}, 14.44 \%$ & $\mathrm{C}_{29}, 16.44 \%$ & $\mathrm{C}_{29}, 15.71 \%$ \\
\hline $\mathrm{C}_{25}, 14.66 \%$ & $\mathrm{C}_{29}, 15.45 \%$ & $\mathrm{C}_{23}, 11.91 \%$ & $\mathrm{C}_{31}, 9.95 \%$ & $\mathrm{C}_{31}, 9.87 \%$ \\
\hline $\mathrm{C}_{23}, 13.47 \%$ & $\mathrm{C}_{27}, 14.47 \%$ & $\mathrm{C}_{25}, 11.57 \%$ & $\mathrm{C}_{30}, 4.11 \%$ & $\mathrm{C}_{30}, 4.83 \%$ \\
\hline
\end{tabular}

- - original data, $"$ - literature data [10] 
For PCA of $P$. peuce populations, six $n$-alkanes of 109 trees of $P$. peuce were selected (according to normal distribution, $\chi^{2}, P \geq 0.05$ and Levene's test, $P \geq 0.05$ ). In this analysis previously examined populations from Montenegro and Serbia were added [9]. PCA revealed that the first two principal axes represent $64.9 \%$ of the total information (Fig. 4). Populations of P. peuce from the Scardo-Pindic mountains (I and II) overlapped and were clearly separated from other populations: Zeletin (III) and Bjelasica (IV) and Mokra Gora (V). $n$-Alkanes which influenced population diversity of $P$. peuce are presented in Table 4.

Cluster analyses. Cluster analyses of populations of both species confirm the separation obtained by PCA (Figs. 5 and 6 ).

It should be noted that needles of $P$. heldreichii and $P$. peuce were sampled in the late summer or early autumn. However, in some literature, the needles of some pines were collected in spring $[8,19,20]$. The range of seasonal variation of Pinus needles is still unknown. Furthermore, the carbon isotopic composition of $n$-alkanes is surely under the influence of environmental factors [22-24].

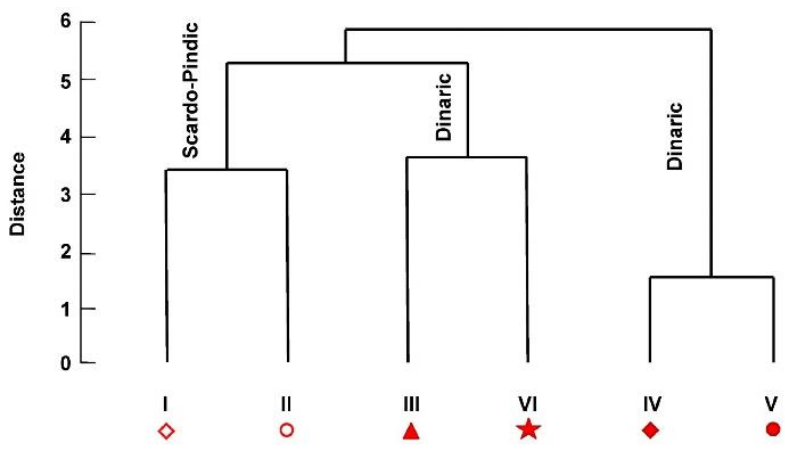

Fig. 5. Dendrogram based on a 'nearest-neighbor method' (square Euclidean distance) of the studied populations I-VI (mean values) of $P$. heldreichii. The numbers on the vertical axis refer to the distance level calculated on the basis of differences between population contents of selected components.

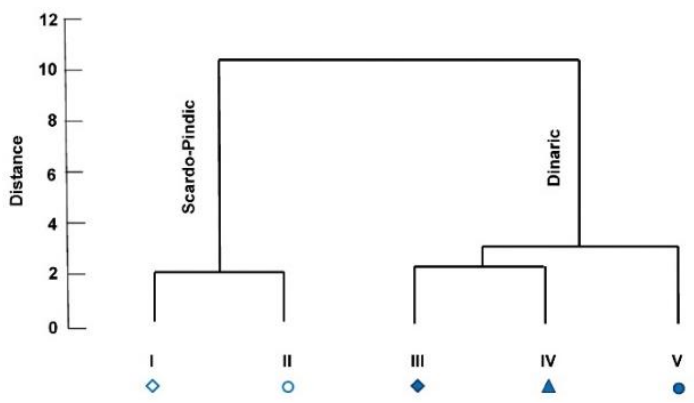

Fig. 6. Dendrogram based on a 'nearest-neighbor method' (square Euclidean distance) of the studied populations I-V (mean values) of $P$. peuce. The numbers on the vertical axis refer to the distance level calculated on the basis of differences between population contents of selected components.

\section{CONCLUSIONS}

The populations of $P$. heldreichii and $P$. peuce from the Dinaric (previous results) and Scardo-Pindic mountains (present data) diverge in terms of $n$-alkane composition. Strong domination of long-chain over mid-chain $n$-alkanes was found in both species. Differences in $n$-alkane range and abundance were confirmed in some other pine species and their varieties [8]. These differences could be conditioned by genetic differentiation and/or the influence of various ecologic factors.

Acknowledgements. This research was supported by a grant from the Ministry of Science and Environmental Protection of Serbia (projects 173029, 172053 and 173011).

\section{REFERENCES}

[1] M. Vidaković, Četinjače. Morfologija i varijabilnost, JAZU i Sveučilišna naklada Liber, Zagreb, 1982.

[2] B. Nikolić, M. Ristić, S. Bojović, V. Matevski, Z. Krivošej, P. D. Marin, Essential-oil composition of the needles collected from natural populations of Macedonian pine (Pinus peuce Griseb.) from the Scardo-Pindic mountain system. Chem. Biodivers., 11, 934-948 (2014). DOI: https://doi.org/10.1002/cbdv.201300343

[3] B. Nikolić, M. Ristić, S. Bojović, Z. Krivošej, V. Matevski, P. D. Marin, Population variability of essential oils of Pinus heldreichii from the Scardo-Pindic Mountains Ošljak and Galičica. Chem. Biodivers., 12, 295-308 (2015). DOI: https://doi.org/10.1002/cbdv.201400135

[4] T. G. Knight, M. A. B. Wallwork, M. Sedgley, Leaf epicuticular wax and cuticle ultrastructure of four Eucalyptus species and their hybrids. Int. J. Plant Sci., 165, 2736 (2004).

DOI: https://www.jstor.org/stable/10.1086/380744

[5] Z. S. Mitić, B. K. Zlatković, S. Č. Jovanović, J. S. Nikolić, B. M. Nikolić, G. S. Stojanović, P. D. Marin, Diversity of needle $n$-alkanes, primary alcohols and diterpenes in Balkan and Charpathian native populations of Pinus nigra J.F. Arnold. Biochem. Syst. Ecol. 80, 46-54 (2018). http://biore.bio.bg.ac.rs/handle/123456789/2914

[6] C. Lutz, V. Heinzmann, P. G. Gülz, Surface structures and epicuticular wax composition of spruce needles after long-term treatment with ozone and acid mist. Environ. Pollut, 64, 313-322 (1990). DOI: https://doi.org/10.1016/0269-7491(90)90053-F

[7] R. S. Dodd, M. M. Poveda, Environmental gradients and population divergence contribute to variation in cuticular wax composition in Juniperus communis. Biochem. Syst. Ecol. 3, 1257-1270 (2003). DOI:10.1016/S0305-1978(03)00031-0

[8] M. Maffei, S. Badino, S. Rossi, Chemotaxonomic significance of leaf wax $n$-alkanes in the Pinales (Coniferales). J. Biol. Res. - Thessalon. 1, 3-19. (2004).

[9] B. Nikolić, V. Tešević, I. Đorđević, M. Jadranin, M, Todosijević, S. Bojović, P.D. Marin, Chemodiversity of nonacosan-10-ol and $n$-alkanes in the needle wax of Pinus 
heldreichii. Chem. Biodivers. 9, 80-90 (2012a), DOI: 10.1002/cbdv.201100179

[10] B. Nikolić, V. Tešević, I. Đorđević, M. Todosijević, M. Jadranin, S. Bojović, P. D. Marin, Population variability of nonacosan-10-ol and n-alkanes in needle cuticular waxes of Macedonian Pine (Pinus peuce Griseb.). Chem. Biodivers. 9, 1155-1165 (2012b). DOI: $10.1002 / \mathrm{cbdv} .201100316$

[11] B. Nikolić, M. Ristić, S. Bojović, V. Matevski, Z. Krivošej, P. D. Marin, Essential-oil composition of the needles collected from natural populations of Macedonian pine (Pinus peuce Griseb.) from the Scardo-Pindic mountain system. Chem. Biodivers. 11, 934-948 (2014). DOI: $10.1002 / \mathrm{cbdv} .201300343$

[12] B. Nikolić, M. Ristić, S. Bojović, P. D. Marin, Population variability of essential oils of Pinus heldreichii from the Scardo-Pindic mountains. Chem. Biodivers. 12, 295-308 (2015). DOI: 10.1002/cbdv. 201400135

[13] M. R. M. Mimura, M. L. F. Salatino, A. Salatino, J. F. A. Baumgratz, Alkanes from epicuticular waxes of Huberia species: taxonomic implications. Biochem. Syst. Ecol. 26,581-588 (1998).

DOI: https://doi.org/10.1016/S0305-1978(97)00131-2

[14] H.Van Den Dool, P. D. Kratz, A generalization of the retention index system including linear temperature programmed gas-liquid partition chromatography. $J$. Chromatogr. 11, 463-471 (1963).

DOI: https://doi.org/10.1016/S0021-9673(01)80947-X

[15] M. A. Mazurek, B. R. T. Simoneit, Caracterization of biogenic and petroleum-derived organic matter in aerosols over remote, rural and urban areas in: Identification and Analysis of Organic Pollutants in Air. (Ed.) Keith, Ann. Arbor, Science / Butterworth Publishers, Boston, 1984, pp. 353-370.

[16] J. Poynter, G. Eglinton, Molecular composition of three sediments from Hole 717C: the Bengal Fan. J. R. Cochran and D.A.V. Stow (Eds), Proc. ODP Sci. Results, 116 , 1990, pp. 155-161.
[17] E. E. Bray, E. D. Evans, Distribution of $n$-paraffins as a clue to recognition of source beds. Geochim. Cosmochim. Acta 22, 2-15 (1961). DOI: https://doi.org/10.1016/0016-7037(61)90069-2

[18] T. K. Kuhn, E. S. Krull, A. Bowater, K. Grice, G. Gleixner, The occurrence of short chain $n$-alkanes with an even over odd predominance in higher plants and soils. Org. Geochem. 41, 88-95 (2010). DOI: https://doi.org/10.1016/j.orggeochem.2009.08.003

[19] D. R. Oros, L. J. Standley, X. Chen, B. R. Simoneit, Epicuticular wax compositions of predominant conifers of western North America. Z. Naturforsch. C. 54, 17-24. (1999). DOI: 10.1515/znc-1999-1-205

[20] Y. Chikaraishi, H. Naraoka, Compound-specific $\delta 2 \mathrm{H}-$ $\delta 13 \mathrm{C}$ analyses of $n$-alkanes extracted from terrestrial and aquatic plants. Phytochemistry 63, 361-371 (2003). DOI: https://doi.org/10.1016/S0031-9422(02)00749-5

[21] B. Nikolić, M. Ristić, S. Bojović, P. D. Marin, Variability of the needle essential oils of Pinus peuce from different populations in Montenegro and Serbia, Chem. Biodivers. 5, 1377-1388 (2009). DOI: http://dx.doi.org/10.1080/11263504.2014.1000999

[22] M. J. Lockheart, I. Poole, P.F.Van Bergen, R.P. Evershed, Leaf carbon isotope composition and stomatal characters: important considerations for palaeoclimate reconstruction. Org. Geochem. 29, 1003-1008 (1998). DOI: https://doi.org/10.1016/S0146-6380(98)00168-5

[23] N. C. Arens, A. H. Jahren, R. Amundson, Can C3 plants faithfully record the carbon isotopic composition of atmospheric dioxide? Paleobiology, 26, 137-164 (2000). DOI: https://doi.org/10.1666/0094-8373(2000)026<0137:C CPFRT $>2.0 . \mathrm{CO} ; 2$

[24] A. Vogts, H. Moossen, F. Rommerskirchen, J. Rullkötter, Distribution patterns and stable carbon isotopic composition of alkanes and alkan-1-ols from plant waxes of African rain forest and savanna $\mathrm{C} 3$ species. Org. Geochem. 40, 1037-1054 (2009).

DOI:10.1016/j.orggeochem.2009.07.011 